\title{
Lessons Learnt in The Management of Acute Complications following Liver Injury in Children
}

\author{
Raghunath B. Vasudeva *, Bahubali D. Gadgade, Praveena D. Lakshmi \\ Department of Pediatric Surgery, Bangalore Medical College, Bengaluru, India
}

\section{Article history:}

Received: March 21, 2020

Revised: May 27, 2020

Accepted: June 10, 2020

\section{${ }^{*}$ Corresponding Author:}

Bahubali D. Gadgade

Department of Pediatric Surgery,

Bangalore Medical College, KR road,

Bengaluru, 560002 India

E-mail: bahudg@gmail.com

\section{ORCID}

Bahubali D. Gadgade

https://orcid.org/0000-0001-7868-7875

Praveena D. Lakshmi

https://orcid.org/000-0002-6329-3432

\section{ABSTRACT}

Purpose: Liver injuries constitute an important cause of hospital admissions, pediatric morbidity, and sometimes mortality. This study was undertaken to assess the spectrum of pediatric liver injuries with special emphasis on the management of acute complications.

Methods: This retrospective study used data from the Department of Pediatric Surgery, Bangalore Medical College, Bengaluru, India, between March 2013 and March 2019. All children between 1-18 years were included in the study $(n=33)$. Children were assessed with relevance to age, sex, mode of injury, hemodynamic stability at admission, need for blood transfusions, surgery, radiological investigations, complications, total number of days of stay in hospital, and mortality.

Results: There were 27 (81.8\%) males and 6 (18.18\%) were females. The most common cause for injury was a road traffic accident (60.6\%). The majority (nearly $65 \%$ ) of pediatric liver injuries were Grade II and III. There were 4 children who had post traumatic complications including 2 pseudoaneurysms, 1 inferior vena cava thrombus, and 1 bile leak with a biloma formation which were managed appropriately.

Conclusion: Timely and appropriate radiological evaluation is required for assessments of complications. The majority of complications can be managed conservatively, and minimally invasive techniques like endoscopic retrograde cholangiopancreatography is helpful in the management of complications.

Keywords: inferior vena cava, thrombus, accidental injury, liver, traffic accident, false aneurysm.

\section{Introduction}

Blunt injury to the abdomen represents one of the important causes for pediatric intensive care unit admissions. Liver is one of the frequently injured organs [1,2]. Common aetiologies include road traffic accidents (RTAs), and falling from a height $[1,2]$. Timely and appropriate radiological assessment is very important in the grading of injuries to indicate the severity management, and prognosis, and in the identification of possible complications. Common complications/sequalae seen following liver trauma include bleeding, hemobilia secondary to bleeding pseudo aneurysms, pulmonary embolism secondary to inferior vena caval thrombosis, and bile leakage secondary to bile duct laceration [3].
This retrospective study was undertaken to assess the pediatric demographic profile, nature of the injuries involved, and management options with special emphasis on complications.

\section{Materials and Methods}

This was a retrospective observational study conducted between March 2013 and March 2019 in the Department of Pediatric Surgery, located in a multidisciplinary specialty public sector hospital.

All children admitted to hospital were first assessed in triage by a multidisciplinary team. This team included the 
Department of Pediatric Surgery, Neurosurgery, Orthopedics, Plastic Surgery, Gynecology. Relevant departments were involved on a case by case basis.

After the initial evaluation and stabilization of the patient, the children were moved to the Pediatric High Dependency Unit for further management under the care of Pediatric surgeons. Appropriate referrals were made to other specialties as and when required.

Data regarding children presenting with blunt trauma abdomen (BTA) and hepatic injuries were reviewed from the hospital registry. Data collected were analyzed with relevance to age, sex, mode of injury, hemodynamic stability at admission, need for blood transfusions, need for surgery, need for radiological interventions, complications, total number of days of stay in hospital, and mortality.

Computerized tomography (CT) images of all patients were reviewed by the radiologist. Grading of liver injuries were reviewed. Injuries were scored according to the Organ Injury Scale described by American Association for the Surgery of Trauma [1].

Protocols related to blood transfusion were reviewed. In children who were on conservative treatment, 12-hour hemoglobin ( $\mathrm{Hb})$ and hematocrit (Hct) was performed until values stabilized. Packed red cell transfusion was administered only if children were hemodynamically unstable with documented $\mathrm{Hb}$ and $\mathrm{Hct}$ less than $9 \mathrm{mg} / \mathrm{dl}$ and 30\%, respectively.

The role of minimally invasive techniques including endoscopic retrograde cholangiopancreatography (ERCP) and open surgery (laparotomy) were reviewed. Parents of all the children were contacted by telephone and a follow up status was obtained by questionnaire. Statistical analysis was not performed in this study because the total number of children was small and the focus was to study the complications associated with liver injury.

Hospital committee ethical approval was consented prior to starting this study.

\section{Results}

A total of 33 children with blunt traumatic liver injury were included in the study, of whom 27 (81.8\%) were males and $6(18.2 \%)$ were females. The youngest child was 1.5 years and eldest child was 17 years (median: 10.5 years). The most common cause of blunt traumatic liver injury was RTAs (60.6\%). Other causes included falling from a height (18.2\%), falling whilst playing (18.2\%), and falling off a wall over the child (3\%; Table 1).

Children with blunt traumatic liver injury Grade II and III constituted the majority (nearly 65\%) of injuries, and were observed mostly in children $>6$ years. Blunt traumatic liver injury Grades IV and V were observed in $30 \%$ of children as depicted in Table 1.

Table 1. Patient characteristics and demographics.

\begin{tabular}{|c|c|}
\hline Variables & $n(\%)$ \\
\hline \multicolumn{2}{|l|}{ Age $(y)$} \\
\hline $0-5$ & $6(18.2)$ \\
\hline $06-10$ & $11(33.3)$ \\
\hline $11-18$ & $16(48.5)$ \\
\hline \multicolumn{2}{|l|}{ Sex } \\
\hline Male & $27(81.8)$ \\
\hline Female & $6(18.2)$ \\
\hline \multicolumn{2}{|l|}{ Mechanism of injury } \\
\hline Road traffic accident & $20(60.6)$ \\
\hline Fall from height & $6(18.2)$ \\
\hline Fall from bicycle/playing & $6(18.2)$ \\
\hline Falling wall & $1(3.0)$ \\
\hline \multicolumn{2}{|l|}{ Injury grade (AAST) } \\
\hline Grade I & $2(6.1)$ \\
\hline Grade II & $12(36.4)$ \\
\hline Grade III & $9(27.3)$ \\
\hline Grade IV & $6(18.1)$ \\
\hline Grade V & $4(12.1)$ \\
\hline Shock & $4(12.2)$ \\
\hline Requirement of blood transfusion & $9(27.3)$ \\
\hline \multicolumn{2}{|l|}{ Associated injuries } \\
\hline Central nervous system (EDH/SDH/contusion) & $2(6.1)$ \\
\hline Respiratory (hemothorax/lung contusion) & $12(36.4)$ \\
\hline Kidney (perinephric hematoma, contusion) & $3(9.1)$ \\
\hline $\begin{array}{l}\text { Musculo skeletal (pelvic fracture, femur } \\
\text { fracture, humerus fracture, rib fracture) }\end{array}$ & $7(21.2)$ \\
\hline Adrenal gland (hematoma) & $2(6.1)$ \\
\hline Spleen (hematoma) & $2(6.1)$ \\
\hline Bowel perforation (colon) & $1(3.0)$ \\
\hline Pancreas (contusion) & $1(3.0)$ \\
\hline \multicolumn{2}{|l|}{ Management } \\
\hline Conservative management & $31(94.0)$ \\
\hline Laparotomy & $1(3.0)$ \\
\hline Complications & 4 \\
\hline Mortality & 0 \\
\hline
\end{tabular}

AAST $=$ American Association for the Surgery of Trauma; SDH = subdural hematoma; $\mathrm{EDH}=$ extradural haematoma. 
There were $87 \%$ of children who had normal blood pressure values at presentation. Blood transfusion was required in 9 (27.3\%) children. The majority of these children had high grade injuries and were also associated with injuries to other organs. The requirement of blood transfusion according to the grade of injury is shown in Table 2.

There were 13 (39.4\%) children who had isolated liver injury, while the remaining 20 (60.6\%) children had associated injuries including pelvic fractures, humeral fracture, extradural hematoma, diffuse axonal injury, pancreas and splenic contusion, renal contusion, and adrenal hematoma. Associated injuries are summarized in Table 1.

The average duration of stay in the hospital was 7.5 days. 93.3\% of children were managed conservatively. One child required a laparotomy for an associated colonic perforation. Mortality was not observed in this series cases.

The follow up period ranged from 1 month to 6 years. Out of the 33 patients, responses were recorded in only 20 (60.6\%) cases. All of these children were asymptomatic according to their parents. All these children had an ultrasound of the abdomen which was normal.

There were 4 children who had complications from liver injury and required further treatment. The first child was a 5-year-old male and had a Grade IV liver injury. He had jaundice, and abdominal distension with pain since Day 2 of admission. Ultrasound revealed pooling in the sub hepatic region (400-500 $\mathrm{mL}$ ) which was possibly blood or bile. Since the jaundice was gradually increasing, a hepatoiminodiacetic acid scan (HIDA) was performed and the results confirmed a biliary leak. Magnetic resonance cholangiopancreatography was suggestive of biloma ( $800 \mathrm{~mL}$; Figures 1 and 2). A pig tail insertion was carried out which drained thick bile. Since the

Table 2. Comparison of blood transfusion rates and average hospital stay.

\begin{tabular}{ccc}
\hline Variable & Our series & Series from Arslan et al [2] \\
\hline Blood transfusion & $n(\%)$ & $\%$ \\
Grade I & 0 & 33 \\
Grade II & $1(8.3)$ & 43 \\
Grade III & $1(11.1)$ & 73 \\
Grade IV & $4(66.6)$ & 89 \\
Grade V & $3(75)$ & 100 \\
Hospital stay (d) & Mean (range) & Mean (range) \\
Grade I & $3.5(2-5)$ & $4.5(3-6)$ \\
Grade II & $5.25(2-8)$ & $5(3-6)$ \\
Grade III & $5.45(5-7)$ & $8(4-6)$ \\
Grade IV & $7.2(4-13)$ & Not specified \\
Grade V & $21.5(4-45)$ &
\end{tabular}

bile leak persisted, ERCP with a common bile duct stenting and sphincterotomy was done which showed contrast extravasation from right hepatic duct opposite to insertion of cystic duct. Abrupt cut off of a branch of left hepatic duct was noted. Following this, the leak settled and the pig tail catheter was removed. The common bile duct stent was removed after 6 weeks. Subsequently, the child is on follow up and doing fine.

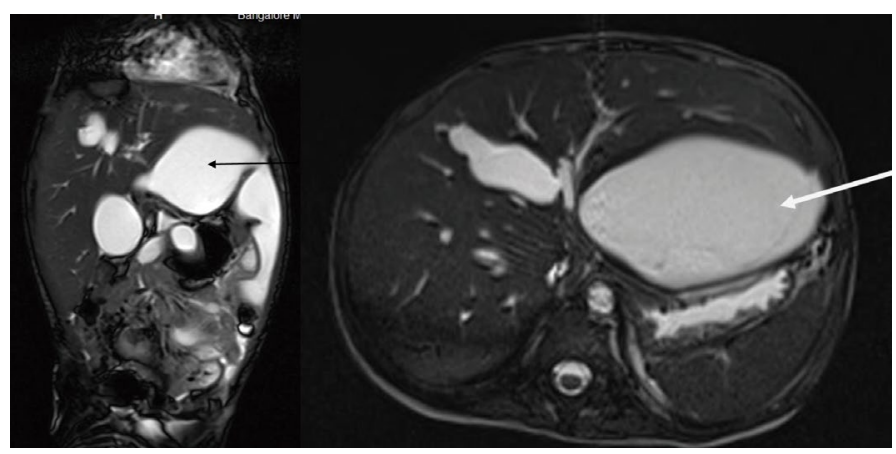

Figure 1. Magnetic resonance imaging scans showing biliary leak with bilioma formation.

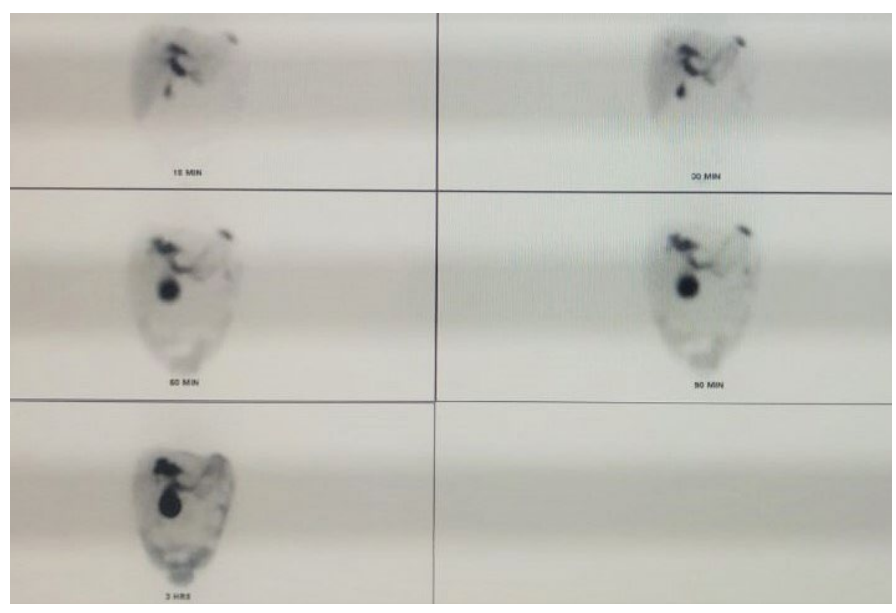

Figure 2. Hepatoiminodiacetic acid scan showing biliary leak.

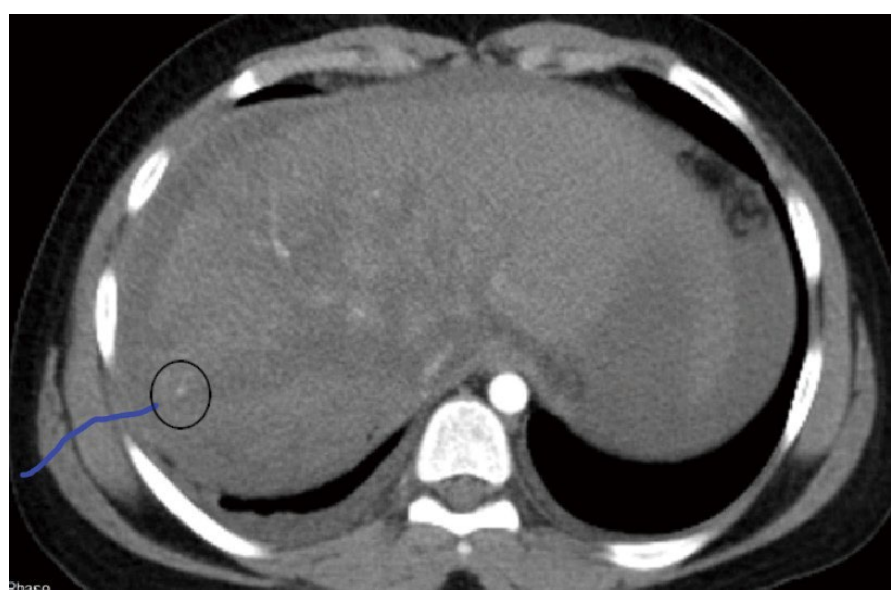

Figure 3. Contrast enhanced computerized tomography scan showing Grade V injury with pseudo aneurysm involving right hepatic artery. 


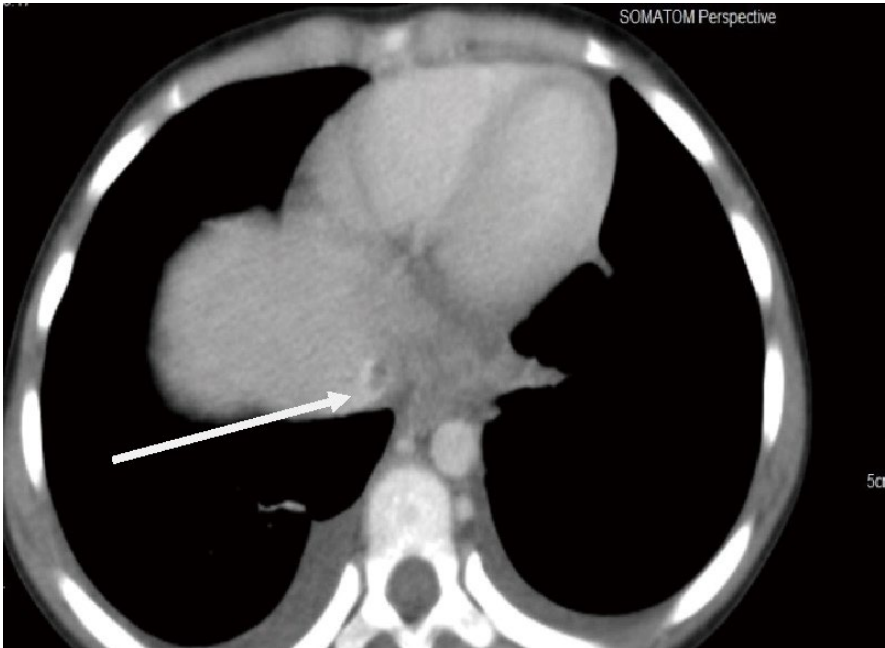

Figure 4. Contrast enhanced computerized tomography scan showing inferior vena cava thrombus.

The second child was a 2-year-old boy, who on evaluation was found to have Grade IV liver injury with a pseudo aneurysm close to the anterior division of the portal vein with bilateral hemothorax. The third child was a 14 -year-old with Grade V liver injury. Evaluation revealed pseudo aneurysm of right hepatic artery in segment 8 (Figure 3). Both the children were treated conservatively, but required 2 aliquots of blood transfusion as they were hemodynamically unstable. Since the bleeding stopped spontaneously, they did not require any further interventions.

The fourth child was an 8-year-old with Grade V liver injury and no vascularity involving the left half of the liver. CT also showed a thrombus involving the supra hepatic vena cava (Figure 4). After hemodynamic stabilization and ruling out coagulopathy, she was started with low molecular weight Heparin and later switched over to oral warfarin for 3 months. Repeat CT done showed resolution of the thrombus.

\section{Discussion}

Blunt trauma abdomen constitutes one of the important causes for hospitalization in children. Most common causes include RTAs and fall from height [2]. Liver is the second most injured organ following BTA [2]. The incidence of complications following liver injury is reportedly low (3-10\%) [3] and includes biliary leak, pulmonary embolism secondary to IVC thrombus, hemobilia secondary to bleeding pseudoaneurysm. Though non operative management (NOM) is the standard of care in a hemodynamically stable child, it is important to identify potential complications [4] and initiate appropriate treatment.

Radiological investigations including ultrasound, contrast enhanced computerized tomography (CECT), and magnetic resonance imaging are the corner stones in evaluation and grading of these injuries. Additional imaging modalities like hepatic iminodiacetic acid scan may occasionally be required.

Traditionally, liver injuries have been graded as per the American Association for the Surgery of Trauma scoring (modified in 2018) [1,5].

In our study, demographic variables with respect to age, sex, etiology of the injury was in coherence with series from other studies [2,4,6,7]. Children with Grade II injuries constituted the majority (36.4\%) followed by Grade III (27.3\%) and Grade IV (18.1\%). Grade I and Grade V injuries were observed in 6\% and $12 \%$ of children, respectively. In a study by Leone et al [8], 13 (49\%) patients had Grade I injury, 9 (33\%) had Grade II injury, 3 (11\%) had Grade III injury, and 2 (7\%) had Grade IV injury. Arslan et al [2] reported 52 children with 6 (12\%) having Grade I, 14 (28\%) having Grade II, 22 (43\%) having Grade III, 9 (17\%) having Grade IV and I (2\%) having Grade V injury.

We did not have any mortality in this series. This may not only indicate the timely and effective care but may also be that more severe cases were dead on arrival before referral to the Department of pediatric surgery. Gross et al [4] reported a mortality rate of $17 \%$ in their series. Of these, $6 \%$ were attributed to liver injury with massive hemorrhage.

Blood transfusion rates in this current study were far lesser compared with the series by Arslan et al [2] as depicted in Table 2. This may be due to the strict guidelines for blood transfusion and stringent monitoring of $\mathrm{Hb} / \mathrm{Hct}$ and hemodynamic stability assessment.

Further, blood transfusions were not required in isolated lowgrade liver injury. Only high-grade liver injury and liver injury associated with other multi organ/system injuries warranted blood transfusions.

The average duration of hospitalization was similar to the series by Arslan et al [2] as shown in Table 2 .

Although the liver is commonly injured following BTA in children, it is usually associated with other organ injuries. In this series, the respiratory system was most commonly involved (36.3\%). The majority of children had rib fractures with hemothorax/lung contusion and of these, only 4 (12.12\%) required inter costal drainage tube insertion. Orthopedic problems including pelvic fractures, radio-ulnar joint dislocation, vertebral fractures were next commonly observed (21.21\%). One of the children had an extradural hematoma which was treated conservatively. Another child had a diffuse axonal injury and was transferred to the Department of Neurology in the Department of Surgy once the liver related issues had been addressed. Surprisingly, there were few splenic injuries.

Hepatic artery pseudo aneurysms secondary to blunt trauma are rare. It is one of the important causes of hemobilia and delayed bleeding [9]. Angiography is the gold standard 
technique for diagnosis of pseudo aneurysms [10]. In this series, we had 2 (6.1\%) children with pseudo aneurysms which were detected by CT scan. Since the children were asymptomatic, they did not require any further diagnostic or therapeutic procedures. Follow up CT scans showed complete resolution of the pseudo aneurysm due to spontaneous thrombosis. Croce et al [11] described the incidence of pseudo aneurysms in children to be around $1.2 \%$. Trans catheter selective embolization of the pseudo aneurysm is the treatment of choice for children who present with hemobilia.

A biliary leak following BTA is quite rare. Although the incidence varies from $1-6 \%$ in adults, the exact incidence in children is not clear [12]. Giss et al [13] reported an incidence of biliary leak as $3.8 \%$ in children treated for blunt liver trauma. Bala et al [14] described a biliary leak rate of $23.9 \%$ in their series. Magnetic resonance cholangiopancreatography is the investigation of choice. It is rare that a HIDA scan and an ERCP may be necessary for diagnosis. Delay in diagnosis could lead to potential morbidity including sepsis, respiratory failure, and abdominal compartment syndrome [15].

Traditionally, biliary leaks were treated by laparotomy with various procedures including primary repair of the bile duct with or without T-tube drainage, partial hepatectomy, and hepatic enterostomy [16,17]. All these procedures had potential morbidity rates because of associated infection/ inflammation, induration of surrounding tissues, and bleeding [17]. In recent years, minimally invasive techniques including ERCP with sphincterotomy/stent placement have made open surgery almost obsolete. Castagnettia et al [18] described varied minimally invasive management approaches in their series including ERCP, laparoscopy, and cholecystostomy. In this series, 1 child with Grade IV injury had a biliary leak from 1 of the branches of the right hepatic duct. ERCP with sphincterotomy and stent placement resolved the biloma.

Inferior vena cava (IVC) thrombosis following hepatic blunt trauma is a rare complication. Proposed theories for thrombosis are extension from hepatic veins, endothelial injury, and pericaval or retro-peritoneal hematoma compressing the IVC [19]. Symptoms resulting from IVC thrombus are nonspecific including vague abdominal/back pain, often attributed to liver injury. Full blown signs of IVC blockage may not be there.

Kim et al [19] suggested that a massive thrombus extending to the supra hepatic region should be treated by emergency thrombectomy and the remaining patients should be treated with IVC filter application with or without anticoagulation therapy. Diab et al [20] recommended a more simplified protocol of considering anticoagulation when not contraindicated with or without placement of an IVC filter on a prophylactic basis. They also recommended that a thrombectomy should be undertaken in cases of massive thrombosis or thrombus progression or clinical decompensation.

In this series, as the parents could not afford a replacement IVC filter, the child was started on anticoagulation therapy to which she responded well. Fortunately, the child did not develop any embolic complications during the treatment period.

\section{Conclusion}

Early detection and appropriate management of post hepatic trauma complications is important. Complications increase with an increasing grade of injury. Requirement for a blood transfusion can be significantly reduced with strict hemodynamic monitoring. Biliary leaks can be successfully managed using ERCP (sphincterotomy) with stenting. An IVC thrombus can be managed by anticoagulation therapy and a thrombectomy is rarely required. Spontaneous resolution of pseudoaneurysms are common and trans catheter embolization is rarely required if it continues to bleed. A multi-disciplinary team is important in the successful management of complex hepatic injuries in children.

\section{Conflicts of Interest}

The authors have no conflicts of interest to declare.

\section{Acknowledgments}

We would like to acknowledge Dr. Anand Alladi, and Dr. S.R. Raghu for helping review the manuscript. I would also like to acknowledge Dr. Deepti for her help in data acquisition.

\section{References}

[1] Moore EE, Cogbill TH, Jurkovich GJ, Shackford SR, Malangoni MA, Champion HR. Organ injury scaling: Spleen and liver. J Trauma 1995;38(3):323-4

[2] Arslan S, Guzel M, Turan C, Doganay S, Dogan AB, Aslan A. Management and treatment of liver injury in children. Ulus Travma Acil Cerrahi Derg 2014;20(1):45-50.

[3] GuanaR, Giulia C,Brunati A, Garofalo S, Schleef J. Biliary complications after Hepatic trauma in children. J Emerg Trauma Shock 2017;10(4):211-4

[4] Gross M, Lynch F, Canty Sr T, Peterson B, Spear R. Management of pediatric liver injuries: A 13-year experience at a pediatric trauma center. J Ped Surg 1999;34(5):811-7.

[5] Kozar RA, Crandall M, Shanmuganathan K, Zarzaur BL, Coburn M, Cribari C, et al. Organ injury scaling 2018 update: Spleen, liver, and kidney. J Trauma Acute Care Surg 2018;85(6):1119-22.

[6] Kepertis C, Zavitsanakis A, Filippopoulos A, Kallergis K. Liver trauma in children: Our experience. J Indian Assoc Pediatr Surg 2008;13(2):61-3

[7] Cooper A, Barlow B, DiScala C, String D. Mortality and truncal injury: The pediatric perspective. J Pediatr Surg 1994;29(1):33-8.

[8] Leone RJ Jr, Hammond JS. Nonoperative management of pediatric blunt 
hepatic trauma. Am Surg 2001;67(2):138-42.

[9] Wani NA, Gojwari TA, Khan NA, Tasleem LK. Hemobilia in a child due to right hepatic artery pseudoaneurysm: Multidetector-row computed tomography demonstration. Saudi J Gastroenterol 2011;17(2):152-4.

[10] Sidhu MK, Shaw DW, Charles PD, John HW, Douglas C. Post-traumatic hepatic pseudoaneurysms in children. Pediatr Radiol 1999;29(1):46-52.

[11] Croce M, Fabian T, Spiers J. Traumatic hepatic artery pseudoaneurysm with hemobilia. Am J Surg 1994;168(3):235-8.

[12] Al-Hassani A, Jabbour G, ElLabib M, Kanbar A, El-Menyar A, Al-Thani. H Delayed bile leak in a patient with grade IV blunt liver trauma: A case report and review of the literature. Int J Surg Case Rep 2015;14:156-9.

[13] Giss SR, Dobrilovic N, Brown RL, Garcia VF. Complications of nonoperative management of pediatric blunt hepatic injury: Diagnosis, management, and outcomes. J Trauma 2006;61(2):334-9.

[14] Bala M, Gazalla SA, Faroja M, Bloom AI, Zamir G, et al. Complications of high grade liver injuries: management and outcomewith focus on bile leaks. Scand J Trauma Resusc Emerg Med 2012;20:20

[15] Aljahdali AH, Murphy JJ. Bile Duct Injury in Children: Is There a Role for Early Endoscopic Retrograde Cholangiopancreatography? Surg J 2018;4(3):e119-22.

[16] Rodriguez-Montes JA, Rojo E, Martin LG. Complications following repair of extrahepatic bile duct injuries after blunt abdominal trauma. World J Surg 2001;25(10):1313-6.

[17] Bade PG, Thomson SR, Hirshberg A, Robbs JV. Surgical options in traumatic injuries of the extrahepatic biliary tract. Br J Surg 1989;76(3):256-8.

[18] Castagnettia M, Houbena C, Patela S, Devlinc J, Harrison P, et al. Minimally invasive management of bile leaks after blunt liver trauma in children. J Pediatr Surg 2006;41(9):1539-44.

[19] Kim KY, So BJ, Park DE. Management of inferior vena cava thrombosis. Korean J Hepatobiliary Pancreat Surg 2014;18(3):97-100

[20] Diab C, Abou Karam A, Laks S, Brunner N. Inferior vena cava thrombus secondary to blunt abdominal trauma. BJR Case Rep 2017;3(3):20160117. 